hep-ph/0503201

\title{
Non-thermal Leptogenesis and a Prediction of Inflaton Mass in a Supersymmetric SO(10) Model
}

\author{
Takeshi Fukuyama ${ }^{1}$, Tatsuru Kikuchi ${ }^{2}$, and Toshiyuki Osaka ${ }^{3}$ \\ Department of Physics, Ritsumeikan University, Kusatsu, Shiga, 525-8577, Japan
}

\begin{abstract}
The gravitino problem gives a severe constraint on the thermal leptogenesis scenario. This problem leads us to consider some alternatives to it if we try to keep the gravitino mass around the weak scale $m_{3 / 2} \sim 100 \mathrm{GeV}$. We consider, in this paper, the non-thermal leptogenesis scenario in the framework of a minimal supersymmetric $\mathrm{SO}(10)$ model. Even if we start with the same minimal $\mathrm{SO}(10)$ model, we have different predictions for low-energy phenomenologies dependent on the types of seesaw mechanism. This is the case for leptogenesis: it is shown that the type-I see-saw model gives a consistent scenario for the non-thermal leptogenesis but not for type-II. The predicted inflaton mass needed to produce the observed baryon asymmetry of the universe is found to be $M_{I} \sim 5 \times 10^{11} \mathrm{GeV}$ for the reheating temperature $T_{R}=10^{6}$ $\mathrm{GeV}$.
\end{abstract}

\footnotetext{
${ }^{1}$ E-mail: fukuyama@se.ritsumei.ac.jp

${ }^{2}$ E-mail: rp009979@se.ritsumei.ac.jp

${ }^{3}$ E-mail: rp006002@se.ritsumei.ac.jp
} 


\section{Introduction}

The supersymmetric (SUSY) grand unified theory (GUT) provides an attractive implication for the understandings of the low-energy physics. In fact, for instance, the anomaly cancellation between the several matter multiplets is automatic in the GUT, since the matter multiplets are unified into a few multiplets, and the experimental data supports the fact of unification of three gauge couplings at the GUT scale, $M_{\mathrm{GUT}}=2 \times 10^{16} \mathrm{GeV}$ assuming the particle contents of the minimal supersymmetric standard model (MSSM) [1, 2]. The right-handed neutrino appeared naturally in the SO(10) GUT provides a natural explanation of the smallness of the neutrino masses through the see-saw mechanism [3], and also the baryon asymmetry of the universe may have its origin in the same dimension-five operator relevant to the neutrino masses through the leptogenesis scenario [4] that would provide a natural explanation for the observed value of the baryon asymmetry [5]

$$
4.9 \times 10^{-11} \leq Y_{B}\left(=\frac{n_{B}}{s}\right) \leq 9.9 \times 10^{-11}(95 \% \text { C.L. }) .
$$

In a series of papers, we have discussed a minimal SO(10) model [6, 7] and its applications to low-energy phenomenologies like neutrino oscillations, neutrinoless double beta decay, leptogenesis [8], lepton flavour violation [9] and proton decay [10]. (As for the minimal $\mathrm{SO}(10)$ models done by the other groups, refer [11.) In these applications, we achieved rather good coincidences with observations. The only exceptional case is leptogenesis where our theory gives over-production of baryon asymmetry in its naive form [8]. In order to relieve this pathology, we were forced to make the second pair of doublets play some roles unlike the conventional case where these doublets are decoupled at the electro-weak scale. However, the so called gravitino problem [12] gives a severe constraint on the reheating temperature, though this problem has been a long history [13] and there are many studies on this subject [14, the recent progress including the hadronic decay processes shed a new doubt on the high reheating temperature $T_{R}$ [15]. If we take it serious and adopt low $T_{R}$, the thermal leptogenesis becomes impossible. This may be good news for us since the minimal SO(10) model in its naive form may survive in the new scenario. In the new scenario is not unique and we consider in this paper non-thermal leptogenesis, where the inflaton decays to the right-handed neutrinos and the successive $B-L$ violating decays of the right-handed neutrinos produce the baryon asymmetry.

\section{Leptogenesis in a minimal SUSY SO(10) model}

Let us first briefly review the conventional leptogenesis scenario [4]. In the following, our discussion is always based on the effective Lagrangian at energies lower than the righthanded neutrino masses such that

$$
\mathcal{L}_{\text {eff }}=-\int d^{2} \theta\left(Y_{\nu}^{i j} N_{i}^{c} L_{j} H_{u}+\frac{1}{2} \sum_{i} M_{R i} N_{i}^{c} N_{i}^{c}\right)+\text { h.c. },
$$


where $i, j=1,2,3$ denote the generation indices, $Y_{\nu}$ is the Yukawa coupling, $L$ and $H_{u}$ are the lepton and the Higgs doublets chiral supermultiplets, respectively, and $M_{R i}$ is the lepton-number-violating mass term of the right-handed neutrino $N_{i}$ (we are working on the basis of the right-handed neutrino mass eigenstates). The peculiar properties of minimal $\mathrm{SO}(10)$ are that we can fix $Y_{\nu}^{i j}$ and $M_{R i}$ unambiguously from the low-energy phenomenologies of quarks and leptons [6, 7].

The lepton asymmetry in the universe is generated by CP-violating out-of-equilibrium decay of the heavy neutrinos, $N \rightarrow \ell_{L} H_{u}^{*}$ and $N \rightarrow \overline{\ell_{L}} H_{u}$. The leading contribution is given by the interference between the tree level and one-loop level decay amplitudes, and the CP-violating parameter is found to be [16]

$$
\epsilon=\frac{1}{8 \pi\left(Y_{\nu} Y_{\nu}^{\dagger}\right)_{11}} \sum_{j=2,3} \operatorname{Im}\left[\left(Y_{\nu} Y_{\nu}^{\dagger}\right)_{1 j}^{2}\right]\left\{f\left(M_{R j}^{2} / M_{R 1}^{2}\right)+2 g\left(M_{R j}^{2} / M_{R 1}^{2}\right)\right\} .
$$

Here $f(x)$ and $g(x)$ correspond to the vertex and the wave function corrections,

$$
\begin{aligned}
f(x) & \equiv \sqrt{x}\left[1-(1+x) \ln \left(\frac{1+x}{x}\right)\right], \\
g(x) & \equiv \frac{\sqrt{x}}{2(1-x)},
\end{aligned}
$$

respectively, and both are reduced to $\sim-\frac{1}{2 \sqrt{x}}$ for $x \gg 1$. So in this approximation, $\epsilon$ becomes

$$
\epsilon=-\frac{3}{16 \pi\left(Y_{\nu} Y_{\nu}^{\dagger}\right)_{11}} \sum_{j=2,3} \operatorname{Im}\left[\left(Y_{\nu} Y_{\nu}^{\dagger}\right)_{1 j}^{2}\right] \frac{M_{R 1}}{M_{R j}} .
$$

Using the Type-I see-saw mass of the neutrino, $M_{\nu}=-Y_{\nu}^{T} M_{R}^{-1} Y_{\nu}\left\langle H_{u}\right\rangle^{2}, \epsilon$ is further written as [17.

$$
\begin{aligned}
\epsilon & =\frac{3}{16 \pi} \frac{M_{R 1}}{\left\langle H_{u}\right\rangle^{2}} \frac{\operatorname{Im}\left[\left(Y_{\nu} M_{\nu}^{*} Y_{\nu}^{T}\right)_{11}\right]}{\left(Y_{\nu} Y_{\nu}^{\dagger}\right)_{11}} \\
& \equiv \frac{3}{16 \pi} \frac{m_{\nu 3} M_{R 1} \delta_{\mathrm{eff}}}{\left\langle H_{u}\right\rangle^{2}} .
\end{aligned}
$$

In the minimal $\mathrm{SO}(10)$ model we have the definite form of $Y_{\nu}$ and estimate these values unambiguously. We have assumed that the lightest $N_{1}$ decay dominantly contributes to the resultant lepton asymmetry. In fact, this is confirmed by numerical analysis in the case of hierarchical right-handed neutrino masses [18]. Using the above $\epsilon$, the generated $Y_{B}$ is described as

$$
Y_{B} \sim \frac{\epsilon}{g_{*}} d,
$$

where $g_{*} \sim 100$ is the effective degrees of freedom in the universe at $T \sim M_{R 1}$, and $d \leq 1$ is so-called the dilution factor. This factor parameterizes how the naively expected value $Y_{B} \sim \epsilon / g_{*}$ is reduced by washing-out processes. 
We can classify the washing-out processes into two cases with and without the external leg of the heavy right-handed neutrinos, respectively. The former includes the inverse-decay process and the lepton-number-violating scatterings mediated by the Higgs boson [19] such as $N+\overline{\ell_{L}} \leftrightarrow \overline{q_{R}}+q_{L}$, where $q_{L}$ and $q_{R}$ are quark doublet and singlet, respectively. The latter case is the one induced by the effective dimension five interaction,

$$
\mathcal{L}_{N}=\frac{1}{2}\left(Y_{\nu}^{T} M_{R}^{-1} Y_{\nu}\right)_{i j}\left(L_{i} H_{u}\right)^{T} C^{-1}\left(L_{j} H_{u}\right)
$$

after integrating out the heavy right-handed neutrinos. This term is nothing but the one providing the see-saw mechanism [3]. The importance of this interaction was discussed in 20, where the interaction was shown to be necessary to avoid the false generation of the lepton asymmetry in thermal equilibrium. While numerical calculations [18 [19 are necessary in order to evaluate the dilution factor precisely, $Y_{B} \sim \epsilon / g_{*}$ roughly gives a correct answer, and the washing-out process is mostly not so effective. Note that this is the consequence from the current neutrino oscillation data as explained in [8].

The lepton asymmetry parameter $\epsilon$ has been evaluated by using the results of the minimal $\mathrm{SO}(10)$ model [7], and results are listed in the following.

\begin{tabular}{|c|c|}
\hline $\tan \beta$ & $|\epsilon|$ \\
\hline \hline 40 & $7.39 \times 10^{-5}$ \\
45 & $6.80 \times 10^{-5}$ \\
50 & $6.50 \times 10^{-5}$ \\
55 & $11.2 \times 10^{-5}$ \\
\hline
\end{tabular}

Unfortunately, the CP-violating parameter $\epsilon$ is too large to be consistent with the observed baryon asymmetry. In order to circumvent this trouble we made use of another pair of $\mathrm{SU}(2)$ doublets appearing in the minimal $\mathrm{SO}(10)$ model. We solved the Boltzman equation and obtained the consistent $Y_{B}$ [8]. However in this case we need the extra Higgs other than those in the MSSM, which may raise the other problems. So it is deserved to consider an alternative solution to this overproduction. On the other hand the gravitino problem forces us low reheating temperature less than the mass of $M_{R 1}$. If we believe it, the above problem becomes fake since thermal $N_{R}$ are not generated in the reheating era. So the minimal $\mathrm{SO}(10)$ model itself drives us the other approaches such as non-thermal leptogenesis scenario [21] or the Affleck-Dine mechanism [22]. In the next section, we discuss on the non-thermal leptogenesis scenario in the minimal SO(10) model.

\section{Non-thermal leptogenesis}

Now we turn to the discussions of the non-thermal leptogenesis scenario [21. In the nonthermal leptogenesis scenario, the right-handed neutrinos are produced through the direct non-thermal decay of the inflaton. 
Here we give a concrete model to specify the inflaton. We add a singlet chiral supermultiplet which plays a role of inflaton $I^{4}$. The interaction Lagrangian relevant for the inflaton and the right-handed neutrinos is given by

$$
\mathcal{L}_{I}=-\frac{1}{2} \int d^{2} \theta\left(M_{I} I^{2}+\sum_{i} \lambda_{i} I N_{i}^{c} N_{i}^{c}\right) .
$$

When inflaton gets a VEV, it gives rise to the Majorana masses for the right-handed neutrinos in addition to the VEV of $(\mathbf{1 0}, \mathbf{1}, \mathbf{3})$ in $\overline{\mathbf{1 2 6}}$ under $S U(4)_{P S} \times S U(2)_{L} \times S U(2)_{R}$ 6. 7]. However, the VEV $\langle I\rangle$ is posted around the GUT scale and $\lambda_{i}$ is found to be $10^{-8}$ later, and this contribution gives a tiny correction to $M_{R}$. Also the first term in Eq. (9) dominates over the second, and is reduced to the chaotic inflationary model 24].

In such a superpotential, the inflaton decay rate is given by

$$
\Gamma\left(I \rightarrow N_{i} N_{i}\right) \simeq \frac{\left|\lambda_{i}\right|^{2}}{4 \pi} M_{I} .
$$

Then the consequently produced reheating temperature is obtained by

$$
T_{R}=\left(\frac{45}{2 \pi^{2} g_{*}}\right)^{1 / 4}\left(\Gamma M_{P}\right)^{1 / 2} .
$$

If the inflaton dominantly couples to $N$, the branching ratio of this decay process is, of course, $\mathrm{BR} \sim 1$. Then the produced baryon asymmetry of the universe can be calculated by using the following formula,

$$
\begin{aligned}
\left(\frac{n_{B}}{s}\right) & =-0.35 \times\left(\frac{n_{N_{1}}}{s}\right) \times\left(\frac{n_{L}}{n_{N_{1}}}\right) \\
& =-0.35 \times \frac{3}{2} \mathrm{BR}\left(I \rightarrow N_{1} N_{1}\right)\left(\frac{T_{R}}{M_{I}}\right) \times \epsilon .
\end{aligned}
$$

With the hierarchical mass spectra for the right-handed neutrinos, it can be approximated as

$$
\left(\frac{n_{B}}{s}\right)=-1.95 \times 10^{-10} \times \mathrm{BR} \times\left(\frac{T_{R}}{10^{6} \mathrm{GeV}}\right)\left(\frac{M_{R 1}}{M_{I}}\right)\left(\frac{m_{\nu 3}}{0.065 \mathrm{eV}}\right) \times \delta_{\mathrm{eff}},
$$

where $\delta_{\text {eff }} \equiv \operatorname{Im}\left[\left(Y_{\nu} M_{\nu}^{*} Y_{\nu}^{T}\right)_{11}\right] /\left[m_{\nu 3}\left(Y_{\nu} Y_{\nu}^{\dagger}\right)_{11}\right]$ denotes the effective value of the CP violating phase parameter relevant for the leptogenesis and it can be estimated as $\delta_{\text {eff }}=-0.166$ in our model. As it can easily be seen that it is possible to produce the baryon asymmetry of the universe by using the reheating temperature as low as, $T_{R} \lesssim 10^{6} \mathrm{GeV}$. Hence, a very wide range of the gravitino mass can be allowed, $m_{3 / 2} \gtrsim 10^{6} \mathrm{MeV}$. The result of the detailed numerical calculation based on Eq. (12) is shown in Fig. 1. As shown in Fig. 1(a) that the

\footnotetext{
${ }^{4}$ There is an alternative scenario in which we regard one of the scalar partners of the right-handed neutrinos as inflaton [23. But in this case, we obtained the reheating temperature $T_{R} \sim 4 \times 10^{12}[\mathrm{GeV}]$ that is too high for the weak scale gravitino. So it is driven by necessity to consider the other possibility like a model presented in this paper if we keep the gravitino mass at the weal scale.
} 
predicted inflaton mass is heavier than the lightest right-handed neutrino $\left(M_{R 1}=1.6 \times 10^{11}\right.$ $\mathrm{GeV}$ ) in our model [7. Hence the non-thermal leptogenesis is well workable. But in model 25], you can see from Fig. 1(b) that the calculated inflaton mass is lighter than the lightest right-handed neutrino mass $\left(M_{R 1}=2.7 \times 10^{13} \mathrm{GeV}\right)$, and the non-thermal leptogenesis scenario is prohibited by the kinematics. We hasten to add that this conclusion is valid under the non-thermal leptogenesis under the gravity mediated SUSY breaking scenario.

It can be read off from Fig. 1(a) that the observed value of the baryon asymmetry leads to the inflaton mass around $M_{I} \sim 5 \times 10^{11} \mathrm{GeV}$. This corresponds to the coupling constant of the inflaton to the right-handed neutrinos as $\lambda_{i} \sim 10^{-8}$. Such a small coupling indicates that the model can naturally fit into the chaotic inflationary model [24] based on a minimal supersymmetric SO(10) model.

\section{Summary}

In confronting with the recent progress on the gravitino problem [15, the usual thermal leptogenesis scenario encounters some problems. In this paper, we have explored the nonthermal leptogenesis as an alternative scenario to the thermal one. We have estimated the baryon asymmetry of the universe based on a minimal supersymmetric $\mathrm{SO}(10)$ model with type-I see-saw mechanism. The result of our analysis shows that the non-thermal scenario well works within the minimal SO(10) model, and we have found the inflaton mass around $M_{I} \sim 5 \times 10^{11} \mathrm{GeV}$ gives the observed value of the baryon asymmetry of the universe. In this analysis, we have used the reheating temperature $T_{R}=10^{6} \mathrm{GeV}$ which was chosen so as to realize the weak scale gravitino mass $m_{3 / 2} \sim 100 \mathrm{GeV}$ without causing the gravitino problem. Even if these values are relaxed by one order of magnitude $\left(m_{3 / 2} \lesssim 10 \mathrm{TeV}, T_{R}=10^{7} \mathrm{GeV}\right)$, the result is still valid.

In this paper, we have assumed that full thermalization occurs soon after the inflation. Although there is a discussion on this point [26], the main motivation of this paper is to give a quantitative estimation for the non-thermal leptogenesis scenario in the minimal $\mathrm{SO}(10)$ model. Therefore, we leave the consideration about thermalization processes for future study.

\section{Acknowledgments}

The work of T.F. is supported in part by the Grant-in-Aid for Scientific Research from the Ministry of Education, Science and Culture of Japan (\#16540269). He is also grateful to Professors D. Chang and K. Cheung for their hospitality at NCTS. The work of T.K. are supported by the Research Fellowship of the Japan Society for the Promotion of Science (\#7336). 


\section{References}

[1] C. Giunti, C. W. Kim and U. W. Lee, Mod. Phys. Lett. A 6, 1745 (1991); P. Langacker and M. x. Luo, Phys. Rev. D 44, 817 (1991); U. Amaldi, W. de Boer and H. Furstenau, Phys. Lett. B 260, 447 (1991).

[2] As early works before LEP experiments, see: S. Dimopoulos, S. Raby and F. Wilczek, Phys. Rev. D 24, 1681 (1981); L. E. Ibañéz and G. G. Ross, Phys. Lett. B 105, 439 (1981); M. B. Einhorn, D. R. Jones, Nucl. Phys. B 196, 475 (1982); W. Marciano, G. Senjanović, Phys. Rev. D 25, 3092 (1982).

[3] T. Yanagida, in Proceedings of the workshop on the Unified Theory and Baryon Number in the Universe, edited by O. Sawada and A. Sugamoto (KEK, Tsukuba, 1979); M. Gell-Mann, P. Ramond, and R. Slansky, in Supergravity, edited by D. Freedman and P. van Niewenhuizen (north-Holland, Amsterdam 1979); R. N. Mohapatra and G. Senjanović, Phys. Rev. Lett. 44, 912 (1980).

[4] M. Fukugita and T. Yanagida, Phys. Lett. B 17445 (1986); For a recent review, see for instance, W. Buchmuller, R. D. Peccei and T. Yanagida, arXiv:hep-ph/0502169.

[5] S. Eidelman et al. [Particle Data Group], Phys. Lett. B 592, 1 (2004).

[6] K. Matsuda, Y. Koide, T. Fukuyama, Phys. Rev. D 64, 053015 (2001),

K. Matsuda, Y. Koide, T. Fukuyama and H. Nishiura, Phys. Rev. D 65, 033008 (2002).

[7] T. Fukuyama and N. Okada, JHEP 0211011 (2002) arXiv:hep-ph/0205066.

[8] T. Fukuyama and N. Okada, Mod. Phys. Lett. A $17 \quad 1725$ (2002) [ArXive:hep-ph/0202214].

[9] T. Fukuyama, T. Kikuchi and N. Okada, Phys. Rev. D 68, 033012 (2003) [ArXive:hep-ph/0304190].

[10] T. Fukuyama, A. Ilakovac, T. Kikuchi, S. Meljanac and N. Okada, JHEP 0409, 052 (2004) arXiv:hep-ph/0406068.

[11] K. S. Babu and R. N. Mohapatra, Phys. Rev. Lett. 70, 2845 (1993). B. Bajc, G. Senjanović and F. Vissani, Phys. Rev. Lett. 90, 051802 (2003) arXiv:hep-ph/0210207; H. S. Goh, R. N. Mohapatra and S. P. Ng, Phys. Lett. B 570, 215 (2003) arXiv:hep-ph/0303055, Phys. Rev. D 68, 115008 (2003) arXiv:hep-ph/0308197; B. Dutta, Y. Mimura and R. N. Mohapatra, Phys. Rev. D 69, 115014 (2004) arXiv:hep-ph/0402113.

[12] S. Weinberg, Phys. Rev. Lett. 48, 1303 (1982). 
[13] M. Y. Khlopov and A. D. Linde, Phys. Lett. B 138 (1984) 265; I. V. Falomkin, G. B. Pontecorvo, M. G. Sapozhnikov, M. Y. Khlopov, F. Balestra and G. Piragino, Nuovo Cim. A 79 (1984) 193 [Yad. Fiz. 39 (1984) 990]; M. Yu. Khlopov, Yu. L. Levitan, E. V. Sedelnikov and I. M. Sobol, Phys. Atom. Nucl. 57 (1994) 1393 [Yad. Fiz. 57 (1994) 1466].

[14] See for instance, L. Roszkowski and R. Ruiz de Austri, arXiv:hep-ph/0408227.

[15] M. Kawasaki, K. Kohri and T. Moroi, arXiv:astro-ph/0402490; Phys. Rev. D 71, 083502 (2005) arXiv:astro-ph/0408426.

[16] L. Covi, E. Roulet, and F. Vissani, Phys. Lett. B 384, 169 (1996); W. Buchmuller and M. Plumacher, Phys. Lett. B 431, 354 (1998).

[17] W. Buchmuller and T. Yanagida, Phys. Lett. B 445399 (1999).

[18] M. Plumacher, Z. Phys. C 74, 549 (1997).

[19] M. A. Luty, Phys. Rev. D 45, 455 (1992).

[20] E. W. Kolb and S. Wolfram, Nucl. Phys. B 172, 224 (1980); J. N. Fry, K. A. Olive, and M. S. Turner, Phys. Rev. D 22, 2953 (1980).

[21] G. Lazarides and Q. Shafi, Phys. Lett. B 258, 305 (1991); K. Kumekawa, T. Moroi, T. Yanagida, Prog. Theor. Phys. 92, 437 (1994); G. F. Giudice, M. Peloso, A. Riotto and I. Tkachev, JHEP 9908, 014 (1999) arXiv:hep-ph/9905242; T. Asaka, K. Hamaguchi, M. Kawasaki, T. Yanagida, Phys. Lett. B 464, 12 (1999), Phys. Rev. D 61083512 (2000); T. Asaka, H. B. Nielsen and Y. Takanishi, Nucl. Phys. B 647, 252 (2002) arXiv:hep-ph/0207023; A. Mazumdar, Phys. Lett. B 580, 7 (2004) arXiv:hep-ph/0308020.

[22] I. Affleck and M. Dine, Nucl. Phys. B 249, 361 (1985).

[23] T. Fukuyama and T. Kikuchi, JHEP 0505, 017 (2005) arXiv:hep-ph/0412373.

[24] A. Linde, Phys. Lett. B 129, 177 (1983).

[25] B. Dutta, Y. Mimura and R. N. Mohapatra, Phys. Rev. D 69, 115014 (2004) arXiv:hep-ph/0402113.

[26] R. Allahverdi and A. Mazumdar, arXiv:hep-ph/0505050. 


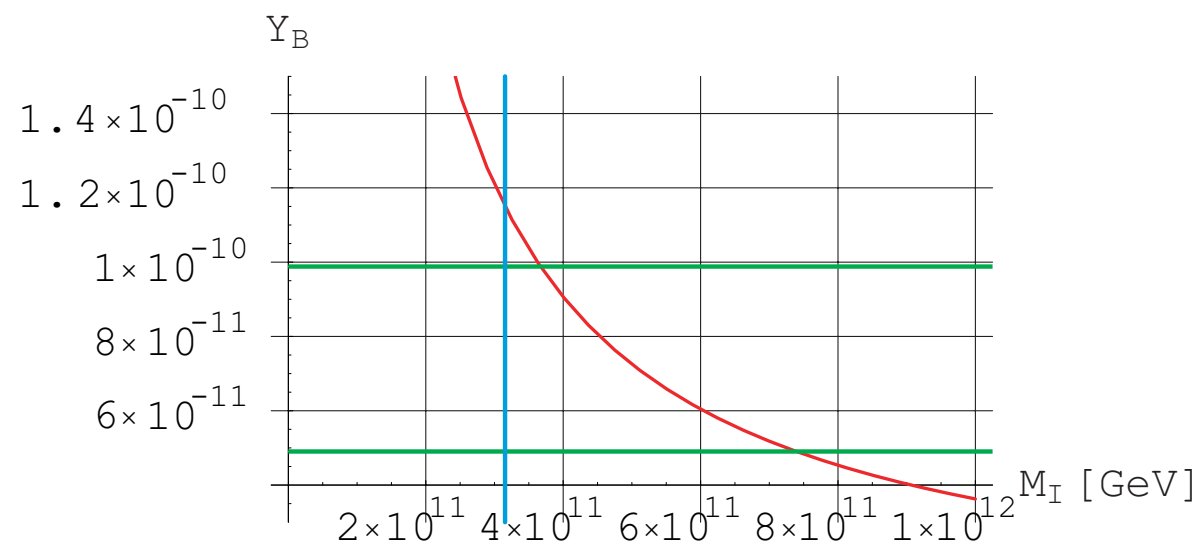

(a) The lepton asymmetry parameter $\epsilon$ has been taken from [7]

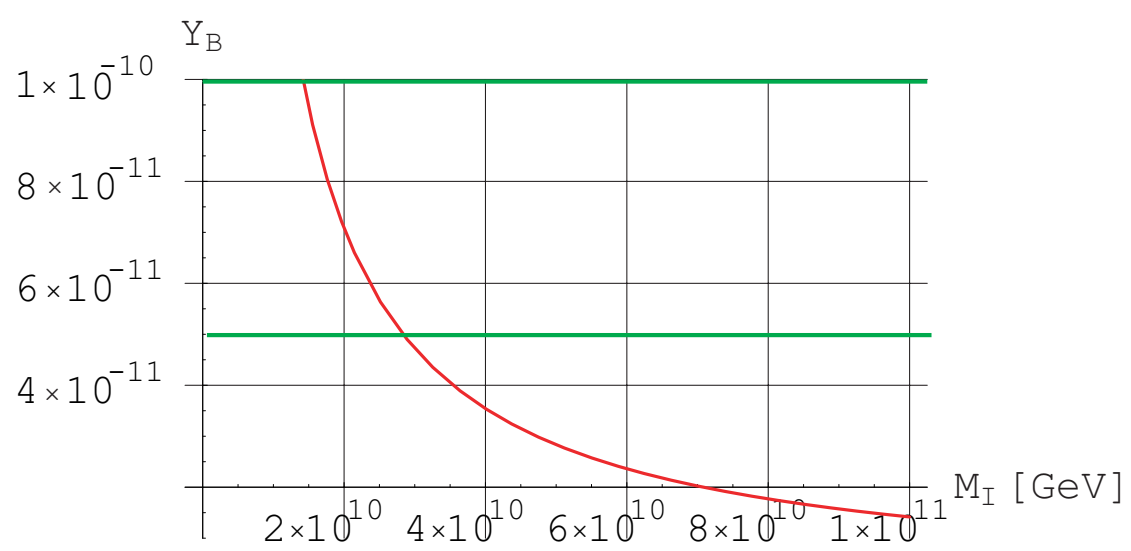

(b) The lepton asymmetry parameter $\epsilon$ has been taken from [25]

Figure 1: The predicted baryon asymmetry of the universe $Y_{B}=n_{B} / s$ as a function of the inflaton mass $M_{I}[\mathrm{GeV}]$ with the reheating temperature $T_{R}=10^{6} \mathrm{GeV}$ and $\mathrm{BR}=1$. In Fig. 1(a), the vertical blue line represents the kinematical cut for the inflaton to have enough energy to decay, $E \geq 2 M_{R 1}$, i.e., the right hand side of this line is allowed from the kinematics. Two horizontal green lines represent the upper and the lower bounds on the observed value of the baryon asymmetry at $95 \%$ C.L. 